\title{
One-Time Acute Heat Treatment Is Effective for Attenuation of the Exaggerated Exercise Pressor Reflex in Rats With Femoral Artery Occlusion
}

\section{Lu Qin and Jianhua Li* \\ Heart and Vascular Institute, Penn State University College of Medicine, Hershey, PA, United States}

OPEN ACCESS

Edited by:

Philip S. Clifford,

University of Illinois at Chicago,

United States

Reviewed by:

Audrey J. Stone,

The University of Texas at Austin,

United States

Bruno Tesini Roseguini,

Purdue University, United States

*Correspondence:

Jianhua Li

jianhuali@pennstatehealth.psu.edu

Specialty section:

This article was submitted to

Exercise Physiology,

a section of the journal

Frontiers in Physiology

Received: 05 May 2020

Accepted: 14 July 2020

Published: 06 August 2020

Citation:

Qin L and Li J (2020) One-Time Acute Heat Treatment is Effective for

Attenuation of the Exaggerated Exercise Pressor Reflex in Rats With

Femoral Artery Occlusion.

Front. Physiol. 11:942.

doi: 10.3389/fphys.2020.00942
The purpose of this study was to determine the effects of one-time acute heat treatment $(\mathrm{HT})$ on the exaggerated exercise pressor reflex in a model of peripheral arterial insufficiency induced by ligation of the femoral artery and was to further examine the underlying mechanism of ATP-P2X $X_{3}$ signal activity during this process. The blood pressure $\mathrm{BP}$ ) response to static muscle contraction and muscle tendon stretch was recorded to determine the exercise pressor reflex. Also, $\alpha \beta$-methylene ATP ( $\alpha \beta$-me ATP) was injected into the arterial blood supply of the hindlimb muscles to stimulate $\mathrm{P}_{2} \mathrm{X}_{3}$ receptors in the muscle afferent nerves. To process one-time acute $\mathrm{HT}$, a heating pad was placed locally on the hindlimb and the muscle temperature $(\mathrm{Tm})$ was increased by $\sim 1.5^{\circ} \mathrm{C}$ and maintained for $5 \mathrm{~min}$. Compared with control rats, a greater mean arterial pressure (MAP) response to muscle contraction was observed in rats with femoral occlusion in a pre-heat control session $(28 \pm 2 \mathrm{mmHg}$ in occluded rats $/ n=12 \mathrm{vs} .18 \pm 2 \mathrm{mmHg}$ in control rats $/ n=9 ; p<0.05)$. The one-time acute HT attenuated the amplification of the BP response in rats with femoral artery occlusion (MAP response: $19 \pm 8 \mathrm{mmHg}$ in occluded rats $+\mathrm{HT} / n=11 ; p<0.05$ vs. occluded rats). In contrast, $\mathrm{HT}$ did not significantly attenuate amplification of MAP response to muscle stretch and $\alpha \beta$-me ATP injection in rats with femoral artery occlusion and controls (all $p>0.05$ ). Our data suggest that one-time acute HT selectively attenuates the amplified pressor response induced by activation of the metabolic and mechanical components of the reflex in rats after femoral artery occlusion. The suppressing effects of acute HT on the exaggerated exercise pressor reflex are likely mediated through a reduction in metabolites (e.g., ATP) stimulating the muscle afferent nerves in contracting muscle, but unlikely through direct alteration of $\mathrm{P} 2 \mathrm{X}$ receptors per se.

Keywords: femoral artery occlusion, muscle temperature, exercise, ATP, blood pressure 


\section{INTRODUCTION}

On a global scale, peripheral artery disease (PAD) is one of the major cardiovascular concerns and affects more than 200 million individuals (Song et al., 2019). The clinical manifestation of intermittent claudication largely jeopardizes the daily mobility performance of PAD patients as it induces pain in legs during the physical activity (McDermott et al., 2004). Supervised exercise therapy has been long considered to be one of the first-line strategies for the treatment and life style management of PAD patients (Stewart et al., 2002; Hamburg and Balady, 2011). However, a risk of cardiovascular events should be considered for the patients during the exercise activity (Collins and Billman, 1989).

In the process of exercise, the mechanical stimuli and the metabolic products (e.g., lactate, ATP, and protons) generated in the contracting muscles activate the receptors in the Group III and IV muscle afferents (Li and Xing, 2012). The activation signals are transferred through the primary sensory dorsal root ganglion (DRG), the dorsal horn of spinal cord, and then projected to cardiovascular nuclei in the brainstem (Mitchell et al., 1983; Kaufman and Forster, 1996). The activation of the cardiovascular nuclei in the brainstem induces increases in the sympathetic nervous activity (SNA) and the consequent amplification of cardiovascular activities. The peripheral mechanism leading to increases in blood pressure (BP) and heart rate (HR) responses to muscle contraction during exercise was termed as the "exercise pressor reflex (EPR)" (Coote et al., 1971; McCloskey and Mitchell, 1972; Victor et al., 1988; Sinoway et al., 1989). The SNA also reduces muscle metabolite-mediated vasodilation (Shoemaker et al., 1999; Hammond et al., 2001), and therefore, limits the blood flow directed to the exercising muscles. The decreasing blood flow in the affected limb partly contributes to the exercise intolerance in PAD patients (Wilson and Mancini, 1993). Meanwhile, a greater increase in arterial $\mathrm{BP}$, which is considered to be attributed to the exaggerated $\mathrm{EPR}$, is more commonly observed in PAD patients than in normal subjects during walking (Baccelli et al., 1999; Bakke et al., 2007). Thus, it is important to study interventions and the underlying mechanisms to alleviate SNA and $\mathrm{BP}$ responses during exercise in PAD.

The ATP-P2 $\mathrm{X}_{3}$ pathway has been considered to play a critical role in regulating EPR during the activation of primary sensory afferents in both healthy and cardiovascular disorders. Our previous studies have demonstrated that (1) compared with the resting status, the interstitial ATP concentration is enhanced when the muscle is active ( $\mathrm{Li}$ et al., 2005), (2) the protein levels of the purinergic $\mathrm{P}_{2} \mathrm{X}_{3}$ expression are increased in the DRG of rats with $24-72 \mathrm{~h}$ of femoral artery occlusion (Liu et al., 2011), and (3) the BP response to stimulation of $\mathrm{P}_{2} \mathrm{X}_{3}$ by $\alpha \beta$-methylene ATP ( $\alpha \beta$-me ATP; P2X $\mathrm{X}_{3}$ agonist) in the afferent nerves is amplified in rats with simulated PAD (Liu et al., 2011; Xing et al., 2013). Meanwhile, it is noted that the functions of P2X receptors have also been reported to be altered by temperature in surrounding tissues (Garcia-Villalon et al., 1997; Ziganshin et al., 2002; Kluess et al., 2005). In a recent study, we demonstrated that the repeated heat exposure protocol [increasing $\sim 1.5^{\circ} \mathrm{C}$ of muscle temperature $(\mathrm{Tm})$ for $30 \mathrm{~min}$, two times per day for three continuous days] effectively suppresses the $\mathrm{P} 2 \mathrm{X}_{3}$ expression and attenuates the $\mathrm{BP}$ response to muscle contraction in rats with simulated PAD (Qin et al., 2020). Nonetheless, it is also necessary to determine a short-period of heating intervention relieving the symptoms of intermittent claudication.

Accordingly, following validating the effectiveness of repeated heating exposure on EPR in the previous study, in this report, we determined whether one-time acute heat treatment $(\mathrm{HT})$ plays an inhibitory role in regulating BP response during activation of the exercise pressor reflex in rats with femoral artery occlusion. We hypothesized that one-time acute HT attenuates amplified BP response to muscle contraction in rats with femoral artery occlusion. We also hypothesized that the alterations in activities of ATP-P2X $\mathrm{X}_{3}$ signal from contracting muscle to sensory nerves mediates this beneficial effect.

\section{METHODS AND MATERIALS}

\section{Animals}

The animal experimental procedures were performed in compliance with the National Institutes of Health Guidelines and were approved by the Institutional Animal Care and Use Committee of the Penn State College of Medicine. Due to the potential effect of female sex hormones on the ATP-P2X pathway (Ma et al., 2011), only male Sprague-Dawley rats (250-350 g; Charles River Laboratory) were used in the present study. Animals were housed in individual cages with free access to standard food and in a temperature-controlled room $\left(25^{\circ} \mathrm{C}\right)$ on a $12-\mathrm{h} / 12 \mathrm{~h}$ light/dark cycle.

\section{Animal Grouping}

The ligation and sham surgery procedures were performed in two sets of rats. Those that underwent the right femoral artery occlusion served as "rats with femoral artery occlusion," whereas those underwent sham surgeries on the right limb served as "control rats." One-time acute HT was performed in some of the control rats and rats with femoral artery occlusion at $72 \mathrm{~h}$ after the surgery. Thus, in the present study four groups of animals were included: control, control $+\mathrm{HT}$, rats with femoral artery occlusion, and rats with femoral artery occlusion + HT.

It is noted that a rat model with femoral artery occlusion does not fully exhibit all the clinical symptoms of PAD. It mimics the critical characteristics of insufficient blood flow, which is commonly observed in PAD (Waters et al., 2004). Thus, in this report, "PAD rats" was used to indicate peripheral arterial insufficiency but not to indicate that the rats had this disease.

\section{Femoral Artery Occlusion}

After being anesthetized with an isoflurane-oxygen mixture (2-5\% isoflurane in $100 \%$ oxygen), femoral artery ligation was performed as previously described (Lu et al., 2013; Xing et al., 2018). In brief, a $\sim 1 \mathrm{~cm}$ surgical incision was made on the skin on right side of groin. After carefully cutting the fascia and removing 
the soft tissue around the veins, the femoral artery was exposed, dissected, and ligated with a surgical suture $\sim 3 \mathrm{~mm}$ distal to the inguinal ligament. For the sham surgery, the same procedures were performed except that a suture was placed below the femoral artery without ligating the artery. Buprenorphine hydrochloride $(0.05 \mathrm{mg} / \mathrm{kg}$, subcutaneously) was administered prior to the surgery for post-operative pain relief. Following the surgery, the animals were kept in the surgery room for $2-3 \mathrm{~h}$ for observation, and then returned to the animal facility.

\section{Tm Monitoring and the One-Time Acute HT Procedure}

Rats were anesthetized with an isoflurane-oxygen mixture (2-5\% isoflurane in $100 \%$ oxygen) and placed on a surgery mat in the prone position. A temperature probe was carefully inserted into the middle point of the gastrocnemius for the continuous monitoring of limb Tm. To elevating the Tm, two heating pads were placed around the examined limb and were removed when the $\mathrm{Tm}$ increased by $\sim 1.5^{\circ} \mathrm{C}$. Thus, during $\mathrm{HT}$, the $\mathrm{Tm}$ was maintained at $\sim 1.5^{\circ} \mathrm{C}$ higher than the baseline. The length of the treatment process was $5 \mathrm{~min}$.

\section{Surgical Preparations to Examine BP Response}

The rats were anesthetized with a mixture of $2-5 \%$ isoflurane and oxygen and ventilated as described previously (Xing et al., 2018). The jugular vein and common carotid artery were cannulated. Fluids were delivered via the jugular vein while a pressure transducer (Model MLT0380, AD Instruments) was connected to the common carotid artery for measurement of the arterial BP via the Powerlab system. HR was calculated beat-to-beat from the arterial pressure pulse using the software of Lab Chart 7 (AD Instruments). To examine P2X-mediated cardiovascular responses, a catheter (PE10) was inserted into the femoral artery for injection of $\alpha \beta$-me ATP. In the rats with femoral artery occlusion, a small incision was made in the femoral artery distal to the previously occluded site. The catheter was then inserted into the artery toward the distal end to deliver the drug into the ischemic limb. During the experiments, baseline BP and fluid balance were maintained with a continuous infusion of saline. The body temperature was maintained at $\sim 37^{\circ} \mathrm{C}$ with a temperature-controlled heat panel (TCAT-2LV, Physitemp Instruments, USA).

Decerebration was performed to eliminate the effects of anesthesia on the reflex pressor response. Prior to the procedure, dexamethasone $(0.2 \mathrm{mg}$, i.v. $)$ was injected to minimize brain stem edema. After decerebration, anesthesia was withdrawn from the rats, and the animals were switched to a ventilator (Model 683, Harvard Apparatus, USA).

A laminectomy procedure (Smith et al., 2001) was performed to expose the lower lumbar and upper sacral portions of the spinal cord and the peripheral ends of the transected L4 and L5 ventral roots were placed on platinum bipolar stimulating electrodes. Experiments were performed 60 min later. Static muscle contractions were induced by electrical stimulation of the L4 and L5 ventral roots (30 s, 3 times motor threshold with a period of $0.1 \mathrm{~ms}$ at $40 \mathrm{~Hz}$ ). In order to activate the mechanical component of the exercise pressor reflex, passive tendon stretch was performed in the right hindlimb. Passive tendon stretch (500 $\mathrm{g}$ of tension) was produced manually over \% $\mathrm{s}$ by using a rack and pinion attached to the Achilles' tendon of rats. Each bout of muscle stretch was maintained for $30 \mathrm{~s}$ after $500 \mathrm{~g}$ of tension was achieved.

\section{Examination of the EPR}

The $\mathrm{BP}$ and $\mathrm{HR}$ responses to muscle contraction and tendon stretch were examined in control, control $+\mathrm{HT}$, PAD, and $\mathrm{PAD}+\mathrm{HT}$. The reflex responses were assessed in three sessions including: 20 min before the one-time acute HT (control session, CON), immediately following the HT (HT session), and $20 \mathrm{~min}$ after the HT (recovery session, REC).

In additional groups, $\mathrm{BP}$ response to arterial injections of $\alpha \beta$-me ATP (0.0625 mM, $0.125 \mathrm{mM}, 0.4 \mathrm{ml} / \mathrm{kg}$ ) was examined in the four groups of rats. The concentrations were selected according to our published work (Liu et al., 2011). The duration of the injection was $1 \mathrm{~min}$ and an interval of $20 \mathrm{~min}$ was allowed between injections. At the end of the experiments, the animals were euthanized by inhalation of an overdose of isoflurane followed by cardiac puncture.

\section{Statistical Analysis}

Unless specified, the data in this study are presented as the mean \pm SD. The SPSS for Windows version 26.0 was utilized for all statistical analyses. Two-way ANOVA was applied to compare the differences in $\mathrm{BP}$ and $\mathrm{HR}$ responses among pre-heat CON, HT, and REC sessions in control rats and PAD rats. As appropriate, post hoc analysis with Tukey's test was applied to compare the differences between specific groups. A $p$ less than 0.05 was considered as statistical significance.

\section{RESULTS}

\section{Tm in the Hindlimb Muscle}

The baseline Tm in control group was significantly higher than that in the PAD group. They were $34.26 \pm 1.37^{\circ} \mathrm{C}$ in control rats vs. $32.81 \pm 1.38^{\circ} \mathrm{C}$ in $\mathrm{PAD}$ rats $(p<0.05$, between control and PAD). With the protocol of $\mathrm{HT}$, the Tm was $35.45 \pm 1.26^{\circ} \mathrm{C}$ in control rats vs. $34.27 \pm 1.39^{\circ} \mathrm{C}$ in $\mathrm{PAD}$ rats $(p<0.05$, between control $+\mathrm{HT}$ and $\mathrm{PAD}+\mathrm{HT})$. Note that the Tm for control rats was $\sim 1.5^{\circ} \mathrm{C}$ higher than that in $\mathrm{PAD}$ rats either before or after HT in this report (Figure 1).

\section{Blood Pressure Response Following Static Muscle Contraction}

No significant differences in basal MAP were found among different heating sessions in the control and PAD groups $(p>0.05$; Table 1). A greater MAP response following static muscle contraction was observed in PAD rats $(26 \pm 8 \mathrm{mmHg}$ in $\mathrm{PAD}$ rats $/ n=11$ vs. $18 \pm 5 \mathrm{mmHg}$ in control rats $/ n=10$; $p<0.05)$ during the pre-heat CON session. The one-time acute HT attenuated the amplification of the BP response in 
PAD rats (MAP response: $19 \pm 8 \mathrm{mmHg}$ in PAD rats $+\mathrm{HT} / n=11 ; p<0.05$ vs. PAD rats). In post-heat REC session (20 min after the HT), a greater MAP response during static muscle contraction was regained in $\mathrm{PAD}$ rats $(27 \pm 3 \mathrm{mmHg}$ in $\mathrm{PAD}$ rats $/ n=10$ vs. $20 \pm 4 \mathrm{mmHg}$ in control rats $/ n=8 ; p<0.05)$. Note that HT did not significantly attenuate the MAP response to muscle contraction in control animals $(18 \pm 7$ in control $+\mathrm{HT}$ vs. $18 \pm 5 \mathrm{mmHg}$ in control; $p>0.05$ between two groups; Figure 2A). There were insignificant differences in the HR response and developed muscle tension during static contraction in different groups and sessions ( $p>0.05$; Figure 2A; Table 2).

\section{Blood Pressure Response Following Muscle Stretch}

No significant differences in basal MAP were found among different heating sessions in the control and PAD groups $(p>0.05$; Table 3). Compared with the control rats, greater

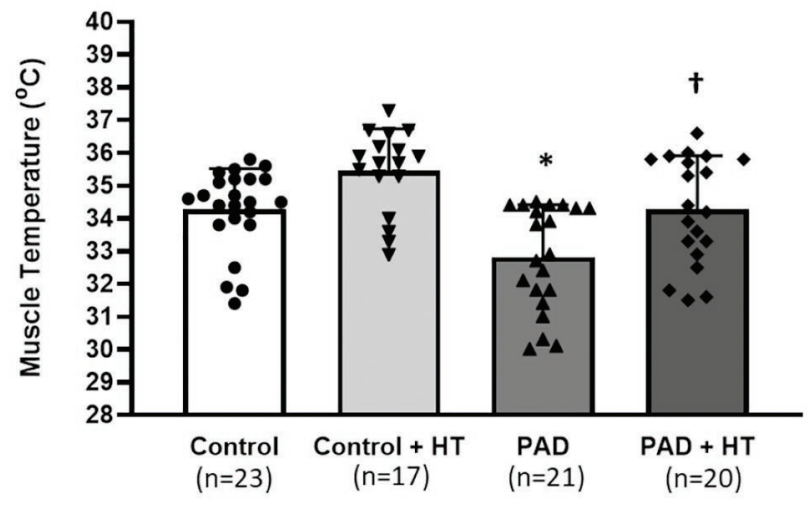

FIGURE 1 | Basal muscle temperature and muscle temperature following the one-time acute heat treatment $(\mathrm{HT})$ in control rats and peripheral artery disease (PAD) rats. Baseline muscle temperature was lower in PAD rats $(n=21)$ than that in control rats $(n=23)$. Following the HT, the muscle temperature was increased by $\sim 1.5^{\circ} \mathrm{C}$. As a result, the muscle temperature was also lower in PAD + HT rats $(n=20)$ than the control $+H T$ rats $(n=17)$. The time length for HT was 5 min. $* p<0.05$ for baseline muscle temperature between control rats and PAD rats; ${ }^{\dagger} p<0.05$ for the muscle temperature following the HT between control $+\mathrm{HT}$ rats and $\mathrm{PAD}+\mathrm{HT}$ rats.

$\mathrm{HT}=$ one-time acute heat treatment. The numbers in the bracket under each group indicate the sample size. Individual data points are indicated for Control

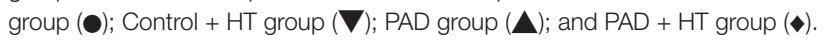

MAP responses during muscle tendon stretch were observed in PAD rats during pre-heat $\mathrm{CON}$ and $\mathrm{HT}$ sessions (pre-heat control session: $21 \pm 4 \mathrm{mmHg}$ in $\mathrm{PAD}$ rats $n=9$ vs. $14 \pm 3 \mathrm{mmHg}$ in control rats $/ n=9$; HT session: $21 \pm 6 \mathrm{mmHg}$ in $\mathrm{PAD}$ rats $/ n=9$ vs. $15 \pm 4 \mathrm{mmHg}$ in control rats $/ n=9$; $p<0.05$ between two groups during two sessions). Likewise, HT had no significant inhibitory effects on the MAP response to muscle stretch in both control and PAD rats, as no significant difference was found in both groups among difference sessions (CON, HT, and REC; $p>0.05$ ). In addition, there were no significant differences in the HR response during muscle stretch in different groups and sessions $(p>0.05$; Figure 2B).

\section{Blood Pressure Response Following $\alpha \beta$-me ATP Injection}

There were no significant difference in the baseline values of MAP among the different heating sessions in control rats and PAD rats $(p>0.05$ between two groups for two dosages of $\alpha \beta$-me ATP; Figure 3; Table 3). Following 0.0625 and $0.125 \mathrm{mM}$ of $\alpha \beta$-me ATP injection, the MAP response in PAD rats was significantly higher than that in control rats during the CON session [0.0625 mM: $32 \pm 5 \mathrm{mmHg}$ in $\operatorname{PAD}(n=9)$, vs. $22 \pm 2 \mathrm{mmHg}$ in control rats $(n=5), p<0.05$ and $0.125 \mathrm{mM}$ : $57 \pm 8 \mathrm{mmHg}$ in PAD rats $(n=7)$ vs. $39 \pm 5 \mathrm{mmHg}$ in control rats $(n=8), p<0.05]$. The greater MAP response to $\alpha \beta$-me ATP remained during HT and REC session. During HT session, the MAP responses induced by $0.0625 \mathrm{mM}$ of $\alpha \beta$-me ATP were $30 \pm 7 \mathrm{mmHg}$ in $\mathrm{PAD}(n=9)$ vs. $21 \pm 5 \mathrm{mmHg}$ in control $(n=5 ; p<0.05$ vs. PAD) and induced by $0.125 \mathrm{mM}$ of $\alpha \beta$-me ATP were $61 \pm 6 \mathrm{mmHg}$ in PAD rats $(n=7)$ vs. $40 \pm 5 \mathrm{mmHg}$ in control rats $(n=8 ; p<0.05$ vs. PAD). During REC session, the MAP response induced by $0.0625 \mathrm{mM}$ of $\alpha \beta$-me ATP was $30 \pm 7 \mathrm{mmHg}$ in $\mathrm{PAD}(n=8)$ vs. $20.93 \pm 4.55 \mathrm{mmHg}$ in control rats $(n=4 ; p<0.05)$ and induced by $0.125 \mathrm{mM}$ of $\alpha \beta$-me ATP was $61 \pm 6 \mathrm{mmHg}$ in PAD rats $(n=6)$ vs. $40 \pm 5 \mathrm{mmHg}$ in control rats $(n=7 ; p<0.05)$. This figure also shows that HT did not attenuate the MAP response to both dosages of $\alpha \beta$-me ATP injection in control rats $(p>0.05$; Figures 3A,B).

\section{DISCUSSION}

In the present study, we demonstrated that the one-time acute $\mathrm{HT}$ attenuated the $\mathrm{BP}$ response to muscle contraction in rats with femoral artery occlusion (Figure 2A), but had no significant

TABLE 1 | The baseline values for blood pressure (BP) in muscle contraction and muscle stretch experiments.

\begin{tabular}{|c|c|c|c|c|c|c|c|}
\hline & & \multicolumn{2}{|c|}{ CON } & \multicolumn{2}{|c|}{ HT } & \multicolumn{2}{|c|}{ REC } \\
\hline & & Control rats & PAD rats & Control rats & PAD rats & Control rats & PAD rats \\
\hline \multirow[t]{2}{*}{ Muscle contraction } & Sample size (N) & 10 & 11 & 9 & 11 & 8 & 10 \\
\hline & $\mathrm{BP}(\mathrm{mmHg})$ & $91 \pm 14$ & $93 \pm 14$ & $95 \pm 16$ & $95 \pm 14$ & $90 \pm 6$ & $96 \pm 13$ \\
\hline \multirow[t]{2}{*}{ Muscle stretch } & Sample size (N) & 9 & 9 & 9 & 9 & 8 & 9 \\
\hline & $\mathrm{BP}(\mathrm{mmHg})$ & $88 \pm 12$ & $92 \pm 17$ & $86 \pm 13$ & $104 \pm 20$ & $89 \pm 14$ & $98 \pm 20$ \\
\hline
\end{tabular}

$B P$, blood pressure; CON, pre-heat control session; HT, one-time acute heat treatment; and REC, post-heat recovery session. No significant differences in basal MAP before contraction and stretch were found among different sessions in the two groups and two different groups in the same session ( $p>0.05)$. All the data were present as mean \pm SD. 
A

Muscle Contraction
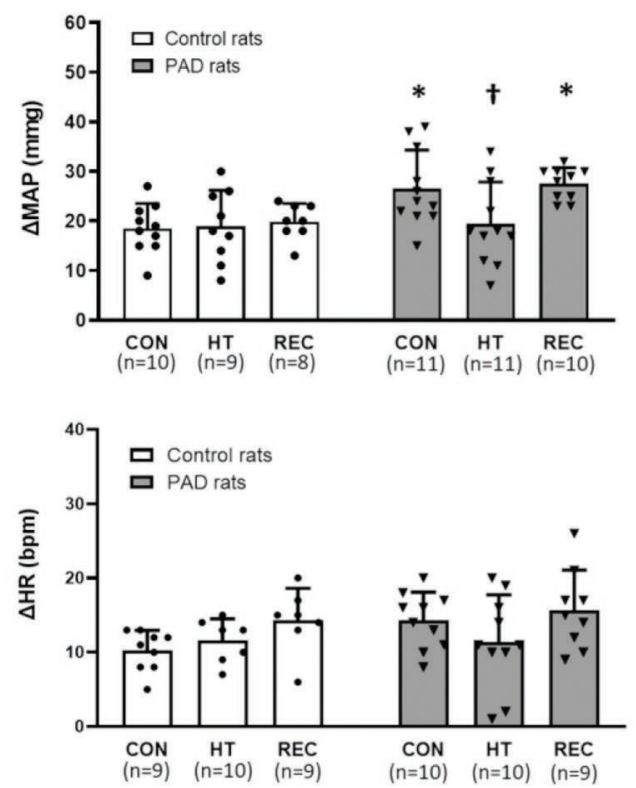

B

Muscle Stretch
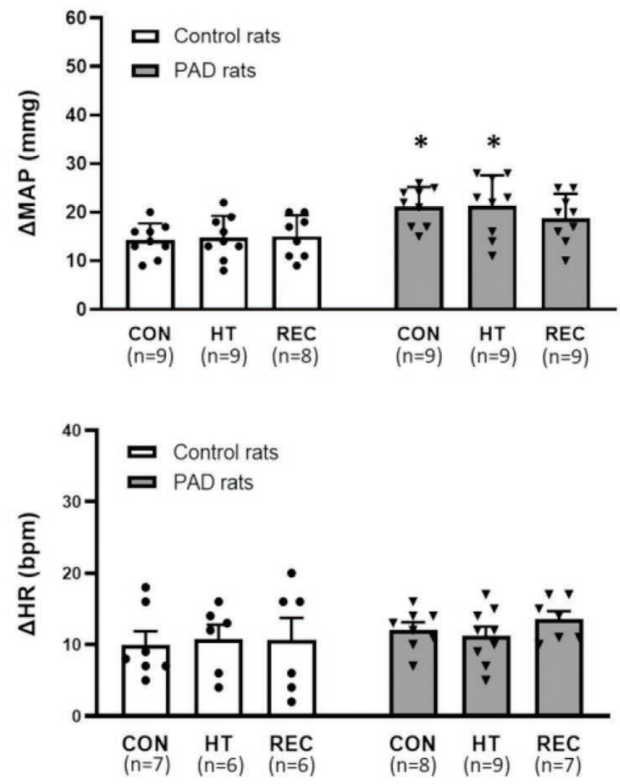

FIGURE 2 | Responses of mean arterial blood (MAP) pressure and heart rate (HR) in control rats and PAD rats induced by muscle contraction and muscle stretch. In control (CON) and recovery (REC) session, MAP response to muscle contraction (A) was amplified in PAD rats when compared with the control rats. In HT session, the MAP response to muscle contraction in PAD rats was lower than that in CON; MAP response to muscle stretch (B) were higher in PAD rats than in control during either $\mathrm{CON}$ and $\mathrm{HT}$ session; ${ }^{*} p<0.05$ vs. control rats and ${ }^{\dagger} p<0.05$ vs. PAD rats in the control session. There were no significant differences in the $\mathrm{HR}$ response during the muscle contraction $\mathbf{( A )}$ and muscle stretch $\mathbf{( B )}$ in different groups and sessions $(p>0.05)$. CON = pre-heat control session; $\mathrm{HT}=$ one-time acute heat treatment; and REC = post-heat recovery session. The numbers in the bracket under each session name indicate the sample size. $\mathbf{0}$ : individual data points for Control rats; $\nabla$ : individual data points for PAD rats.

TABLE 2 | Developed muscle tension during contraction in different groups and sessions.

\begin{tabular}{|c|c|c|c|c|c|c|}
\hline & \multicolumn{2}{|c|}{ CON } & \multicolumn{2}{|c|}{ HT } & \multicolumn{2}{|c|}{ REC } \\
\hline & Control rats & PAD rats & Control rats & PAD rats & Control rats & PAD rats \\
\hline Sample size $(N)$ & 9 & 11 & 8 & 11 & 7 & 10 \\
\hline Tension (g) & $557 \pm 104$ & $485 \pm 105$ & $501 \pm 84$ & $560 \pm 130$ & $604 \pm 104$ & $575 \pm 75$ \\
\hline
\end{tabular}

CON, pre-heat control session; HT, one-time acute heat treatment; REC, post-heat recovery session; and g, gram. No significant differences in muscle tension among different sessions in the two groups and two different groups in the same session $(p>0.05)$. All the data were present as mean $\pm S D$.

effects on the reflex BP response to either the passive muscle stretch or the $\alpha \beta$-me ATP injection (Figures $2 \mathbf{B}, 3 \mathbf{A}, \mathbf{B}$ ).

The role of $\mathrm{ATP}-\mathrm{P} 2 \mathrm{X}_{3}$ pathway in the regulation of EPR in PAD has been studied previously ( $\mathrm{Li}$ et al., 2005; Liu et al., 2011; Xing et al., 2013). During muscle contraction, ATP utilization is accelerated to meet the energy demand. Under ischemia, the production of reactive oxidative species (ROS) inhibits ATPase activity in the mitochondria, and therefore, reduces intracellular ATP utilization (Dobrota et al., 1999; Simao et al., 2011; Jeong et al., 2013). Apart from the suppressed intracellular ATP utilization, during the ischemia condition, the muscular cells are swollen, and thus, the permeability and efflux of the cytoplasmic soluble molecules including ATP is increased (Boudreault and Grygorczyk, 2002). With the increasing amount of intracellular ATP and the enhanced permeability of the ischemic cell membrane, it can be deduced that the interstitial ATP is enhanced during the muscle ischemia and elicits a greater stimulation on the P2X receptors (receptors for ATP) in the muscle afferent nerves. Among the P2X receptors, $\mathrm{P}_{2} \mathrm{X}_{3}$ subtype, specifically, is presented in the primary sensory neurons in DRG (Chen et al., 1995). The elevated ATP concentration in the muscle interstitium enhances the expression and function of $\mathrm{P}_{2} \mathrm{X}_{3}$ and the subsequent EPR response is exaggerated. Consistent with previous studies (Liu et al., 2011; Qin et al., 2020), the BP response following both muscle contraction and muscle stretch was higher in rats with femoral artery occlusion than that in control rats (Figures 2A,B). $\mathrm{BP}$ response to stimulation of $\mathrm{P}_{2} \mathrm{X}_{3}$ by injection of $\alpha \beta$-me ATP, as a part of EPR activation, was also amplified in PAD rats (Figures 3A,B).

It has been suggested that an exaggerated SNA response to exercise in cardiovascular diseases lowers the ventricular 
A

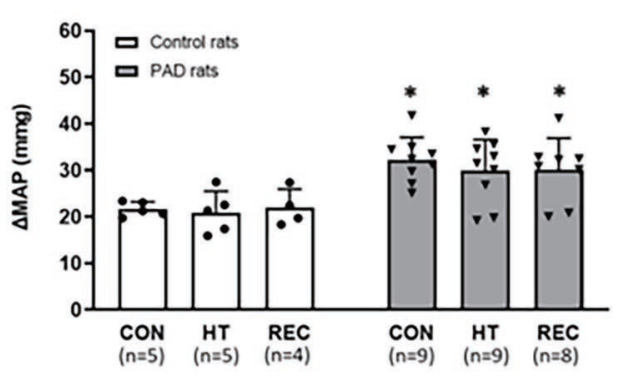

B

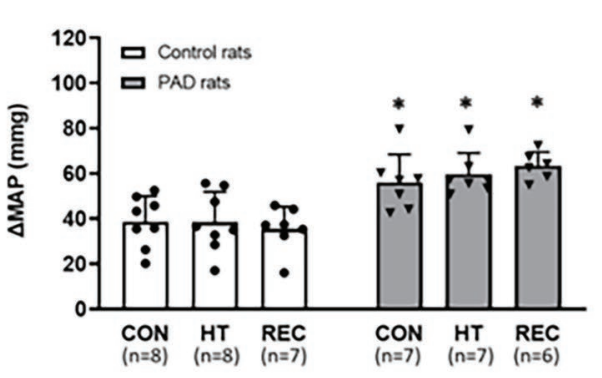

FIGURE 3 | Responses of MAP in control rats and PAD rats following $0.0625 \mathrm{mM}$ (A) and $0.125 \mathrm{mM}$ (B) of $\alpha \beta$-methylene ATP ( $\alpha \beta$-me ATP) administration. In three sessions of CON, HT and REC, MAP response to $0.0625 \mathrm{mM}$ and $0.125 \mathrm{mM}$ of $\alpha \beta$-me ATP administration were higher in PAD rats than in control rats. However, the MAP response was not attenuated in either PAD and in control rats following the one-time acute $\mathrm{HT}$. $* p<0.05$ vs. control rats. CON $=$ pre-heat control session; $\mathrm{HT}=$ one-time acute heat treatment; and REC = post-heat recovery session. The numbers in the bracket under each session name indicate the sample size. individual data points for Control rats; $\nabla$ : individual data points for PAD rats.

TABLE 3 | The baseline values for BP in 0.0625 and $0.125 \mathrm{mM} \alpha \beta$-me ATP administration experiments.

\begin{tabular}{|c|c|c|c|c|c|c|c|}
\hline & & \multicolumn{2}{|c|}{ CON } & \multicolumn{2}{|c|}{ HT } & \multicolumn{2}{|c|}{ REC } \\
\hline & & Control rats & PAD rats & Control rats & PAD rats & Control rats & PAD rats \\
\hline \multirow[t]{2}{*}{$0.0625 \mathrm{mM}$} & Sample size (N) & 5 & 9 & 5 & 9 & 4 & 8 \\
\hline & $\mathrm{BP}(\mathrm{mmHg})$ & $96 \pm 23$ & $99 \pm 21$ & $93 \pm 25$ & $95 \pm 20$ & $99 \pm 24$ & $93 \pm 16$ \\
\hline \multirow[t]{2}{*}{$0.125 \mathrm{mM}$} & Sample size (N) & 8 & 7 & 8 & 7 & 7 & 6 \\
\hline & $\mathrm{BP}(\mathrm{mmHg})$ & $93 \pm 17$ & $90 \pm 20$ & $89 \pm 35$ & $90 \pm 14$ & $91 \pm 14$ & $88 \pm 6$ \\
\hline
\end{tabular}

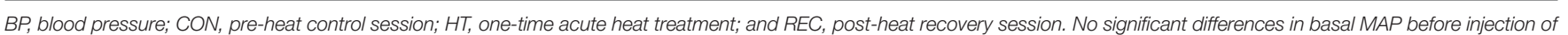
each dosage of $\alpha \beta$-me ATP were found among different sessions in the two groups and two different groups in the same session ( $p>0.05)$. All the data were present as mean \pm SD.

fibrillation threshold, and therefore, increases the probability of cardiovascular risk (e.g., fatal arrhythmias) in patients (Collins and Billman, 1989; Vanoli and Schwartz, 1990). HT, as an inexpensive and non-invasive intervention strategy, has been considered as one of the promising interventions to attenuate the $\mathrm{BP}$ response in the PAD patients. It was reported in an early study that, by elevating the intramuscular temperature from $\sim 32.2$ to $\sim 41.5^{\circ} \mathrm{C}$, action potential amplitude in the sensory nerves was significantly reduced by $32-84 \%$ and the duration reduced by $23-36 \%$ (Rutkove et al., 1997). This result suggested that there might be a negative association between the muscular temperature and the activity of the primary muscle afferent nerves during static exercise. To date, there are a few clinical studies to investigate one-time acute effect of HT on the BP response in PAD patients. For instance, a previous study by Neff et al. (2016) revealed that increasing leg skin temperature to $39-40^{\circ} \mathrm{C}$ for $90 \mathrm{~min}$ significantly attenuated both systolic and diastolic BP in PAD patients with intermittent claudication. Immersion of the lower limbs in water at $42^{\circ} \mathrm{C}$ for $30 \mathrm{~min}$ also decreased arterial BP in PAD patients (Thomas et al., 2017). With the approach of ventral root stimulation in the current study, it was observed that the BP response to the skeletal muscle contraction was enhanced in PAD rats when compared with the control rats. This result was also consistent with what observed in the previous studies (Liu et al., 2011; Qin et al., 2020).
In addition, the exaggerated $\mathrm{BP}$ response was attenuated following increasing $\mathrm{Tm}$ by $\sim 1.5^{\circ} \mathrm{C}$ (Figure $2 \mathrm{~A}$ ).

One explanation for the $\mathrm{HT}$-attenuated $\mathrm{BP}$ response following the muscle contraction is improvement of suppressed ATPase activity in the skeletal muscle of PAD rats. It has been reported that the change of tissue temperature alters cellular enzyme activity. For instance, intrinsic ATPase activity (Paudel and Carlson, 1991) in the New Zealand rabbit skeletal muscle is gradually increased from 0 to $30^{\circ} \mathrm{C}$ (one-fold increase) and dramatically accelerated from 30 to $40^{\circ} \mathrm{C}$ (approximately six-fold increase). In contrast, in our present study (Figure 1), the average baseline $\mathrm{Tm}$ was $34.26 \pm 1.37^{\circ} \mathrm{C}$ in control rats and $32.81 \pm 1.38^{\circ} \mathrm{C}$ in $\mathrm{PAD}$ rats. After the $\mathrm{HT}$, the average terminal Tm was $35.45 \pm 1.26^{\circ} \mathrm{C}$ in control $+\mathrm{HT}$ rats and $34.27 \pm 1.39^{\circ} \mathrm{C}$ in $\mathrm{PAD}+\mathrm{HT}$ rats. These temperatures were within the range from 30 to $40^{\circ} \mathrm{C}$. This suggests that, within that range, the $\mathrm{Tm}$ increment of $\sim 1.5^{\circ} \mathrm{C}$ likely amplified the activity of ATPase in the skeletal muscle in the current study. Moreover, a human study showed that the elevation of Tm was associated with increased ATP turnover rates during the exercise (Gray et al., 2006).

The inhibitory effects of HT on the BP response induced by passive muscle stretch were not observed in PAD rats. It could be hypothesized that muscle stretch stimulated only the mechanoreceptors in the muscle afferent nerves, and a short session of HT was unlikely to affect the process of mechanical activation. 
However, muscle contraction induces an increase in muscle metabolic products and thereby stimulates on both the metaboand mechano-receptors in muscle afferents in evoking the reflex BP response ( $\mathrm{Li}$ and Xing, 2012). As a result, there was likely less ATP released into the interstitial space of muscle during the muscle contraction in PAD rats following this short-time HT protocol. Therefore, there was also a smaller subsequent BP response seen in the current study. In addition, the attenuating effects of $5 \mathrm{~min}$ of $\mathrm{HT}$ on the BP response to muscle contraction were abolished when the Tm was recovered during the recovery session. This suggests that the BP attenuation was a temporary process since the Tm was not elevated during the recovery session.

In our previous study (Qin et al., 2020), using a repeated heating exposure protocol, overexpression of $\mathrm{P}_{2} \mathrm{X}_{3}$ protein in the DRG was attenuated in PAD rats following three-day heating treatment. As a consequence, the BP response to $\alpha \beta$-me ATP injection in the arterial blood supply of the hindlimb muscles was attenuated following that heating protocol. In contrast, 5 min of HT did not attenuate the exaggerated BP response to arterial injection of $\alpha \beta$-me ATP in rats with femoral artery occlusion (Figures 3A,B). This suggests that this short HT protocol did not alter protein expression of $\mathrm{P} 2 \mathrm{X}_{3}$, and thus, the amplitude of $\mathrm{P} 2 \mathrm{X}_{3}$ response stimulated by the $\alpha \beta$-me ATP was unchanged following the HT. In a previous study (Tsuzuki et al., 2001), $\mathrm{P} 2 \mathrm{X}_{3}$ messenger RNA (mRNA) expression was examined following peripheral nerve injury, demonstrating that $\mathrm{P}_{2} \mathrm{X}_{3}$ mRNA in the DRG was enhanced from day 1 post-injury. Our previous study (Liu et al., 2011) also indicated that the $\mathrm{P}_{2} \mathrm{X}_{3}$ expression in the DRG was not upregulated at $6 \mathrm{~h}$ but started to increase $24 \mathrm{~h}$ following the femoral artery ligation. Likewise, the attenuation of the exaggerated $\mathrm{P}_{2} \mathrm{X}_{3}$ expression in the DRG of PAD rats also requires a time course, as shown that there was $3 \mathrm{~h}$ of accumulated heating exposure in the previous repeated heating protocol (Qin et al., 2020). Moreover, during the repeated heating treatment, half of the heating procedures were already completed during the first $24 \mathrm{~h}$ following the ligation, which was before $\mathrm{P} 2 \mathrm{X}_{3}$ upregulation. In contrast, 5 min of HT was unlikely to attenuate the protein $\mathrm{P}_{2} \mathrm{X}_{3}$ overexpression in rats with femoral artery occlusion, which explains why $5 \mathrm{~min}$ of $\mathrm{HT}$ failed to attenuate BP response to $\alpha, \beta$-me-ATP. However, a likelihood cannot be ruled out that $\mathrm{HT}$ could alter the sensitivity of $\mathrm{P} 2 \mathrm{X}_{3}$ receptors on the peripheral endings of afferents, and thus suggests involvement of the reflex $\mathrm{BP}$ response. In addition, without contraction the intracellular ATP failed to efflux into the interstitial space of resting muscle during $\alpha \beta$-me ATP injection. Therefore, rather than stimulated by the ATP released into the interstitial space by muscle contraction, the $\mathrm{P}_{2} \mathrm{X}_{3}$ in the primary sensory afferent were stimulated by injection of $\alpha \beta$-me ATP per se. This somewhat explains why in the current study the higher BP response to $\alpha \beta$-me ATP injection remained in rats with femoral artery occlusion following HT.

In the present study, acute $\mathrm{HT}$ attenuated the $\mathrm{BP}$ response to static contraction, but it did not attenuate the $\mathrm{BP}$ response to respective activation of muscle mechnoreflex and $\mathrm{P}_{2} \mathrm{X}_{3}$ receptors. It seems that the role of metabolites as a mechanism for HT to change BP response to contraction cannot be explained by results of the $\alpha \beta$ me ATP injection. Thus, the metabolic component of the exercise pressor reflex was responsible for the attenuation of the $\mathrm{BP}$ response to static contraction following acute HT cannot be evidently concluded until some other metabolites are identified in engagement of acute HT. On the other hand, muscle contraction led to the release of ATP into the interstitial space. With the enhancement of intracellular ATPase activity following the HT, the amount of ATP released into the interstitial space during muscle contraction was likely less, and therefore, the subsequent BP response was diminished.

In conclusion, one-time acute HT attenuates the amplified pressor response induced by static muscle contraction in rats with femoral artery occlusion, but not by muscle stretch and by stimulation of $\mathrm{P} 2 \mathrm{X}$ in muscle afferents. The suppressing effects of one-time acute elevation of $\mathrm{Tm}$ on the exaggerated exercise pressor reflex in PAD are likely through reducing the activity of temperature sensitive muscle metabolites (e.g., ATP, lactate, and proton) in contracting muscle thereby leading to attenuation of the reflex. Nonetheless, P2X expression and/or responsiveness in muscle sensory nerves might not be altered during the one-time acute HT per se. Whether or not the sensitivity of $\mathrm{P} 2 \mathrm{X}$ receptors on the peripheral endings of afferents is altered during HT and thereby affecting the exercise pressor reflex in PAD needs to be determined.

\section{PERSPECTIVE}

In the present study, static muscle contraction and muscle tendon stretch were used to evoke the exercise pressor reflex in a rat model of femoral artery occlusion. Of note, static exercise protocols are not surrogates for dynamic exercise in humans or treadmill exercise in rats. Therefore, caution should be taken when extrapolating the findings of the current study to whole-body dynamic exercise. The neural mechanisms involved in the effects of acute HT on the exercise pressor reflex still need to be examined using the dynamic exercise models. Additionally, future studies will need to investigate the effects of acute HT on the exercise pressor reflex in females.

The experiments were conducted 3 days following femoral artery occlusion. It could be argued that HT should be tested when the natural compensation to arterial occlusion has stabilized, in part, because the mechanisms mediating initial responses to acute occlusion may differ from those involved in later stages of adaptation (Ziegler et al., 2010). Indeed, the muscle metabolic and inflammatory milieu are largely disturbed in the first few days after the occlusion, creating a scenario that does not mimic the human condition. The physiological responses to exercise in this model are likely exacerbated in the first few days after femoral artery ligation. That is, Prior et al. (2004) reported that in rats BP response during treadmill exercise was greater within 2 days after femoral artery ligation than that after 25 days. It is therefore important to recognize that the reported effects of acute heat treatment may be different, if the experiments are conducted when natural compensation has stabilized. 
DATA AVAILABILITY STATEMENT

All datasets presented in this study are included in the article/ supplementary material.

\section{ETHICS STATEMENT}

The animal protocol was reviewed and approved by the Institutional Animal Care and Use Committee of the Penn State University College of Medicine.

\section{AUTHOR CONTRIBUTIONS}

QL processed cardiovascular response experiments for the drug administration, contributed to the analysis of cardiovascular

\section{REFERENCES}

Baccelli, G., Reggiani, P., Mattioli, A., Corbellini, E., Garducci, S., and Catalano, M. (1999). The exercise pressor reflex and changes in radial arterial pressure and heart rate during walking in patients with arteriosclerosis obliterans. Angiology 50, 361-374. doi: 10.1177/000331979905000502

Bakke, E. F., Hisdal, J., Jorgensen, J. J., Kroese, A., and Stranden, E. (2007). Blood pressure in patients with intermittent claudication increases continuously during walking. Eur. J. Vasc. Endovasc. Surg. 33, 20-25. doi: 10.1016/j. ejvs.2006.06.023

Boudreault, F., and Grygorczyk, R. (2002). Cell swelling-induced ATP release and gadolinium-sensitive channels. Am. J. Physiol. Cell Physiol. 282, C219-C226. doi: $10.1152 /$ ajpcell.00317.2001

Chen, C. C., Akopian, A. N., Sivilotti, L., Colquhoun, D., Burnstock, G., and Wood, J. N. (1995). A P2X purinoceptor expressed by a subset of sensory neurons. Nature 377, 428-431. doi: 10.1038/377428a0

Collins, M. N., and Billman, G. E. (1989). Autonomic response to coronary occlusion in animals susceptible to ventricular fibrillation. Am. J. Physiol. Heart Circ. Physiol. 257, H1886-H1894. doi: 10.1152/ajpheart.1989.257.6.H1886

Coote, J. H., Hilton, S. M., and Perez-Gonzalez, J. F. (1971). The reflex nature of the pressor response to muscular exercise. J. Physiol. 215, 789-804. doi: 10.1113/jphysiol.1971.sp009498

Dobrota, D., Matejovicova, M., Kurella, E. G., and Boldyrev, A. A. (1999). $\mathrm{Na} / \mathrm{K}-\mathrm{ATPase}$ under oxidative stress: molecular mechanisms of injury. Cell. Mol. Neurobiol. 19, 141-149. doi: 10.1023/A:1006909927287

Garcia-Villalon, A. L., Padilla, J., Monge, L., Fernandez, N., Gomez, B., and Dieguez, G. (1997). Role of the purinergic and noradrenergic components in the potentiation by endothelin-1 of the sympathetic contraction of the rabbit central ear artery during cooling. Br. J. Pharmacol. 122, 172-178. doi: $10.1038 /$ sj.bjp.0701359

Gray, S. R., De Vito, G., Nimmo, M. A., Farina, D., and Ferguson, R. A. (2006). Skeletal muscle ATP turnover and muscle fiber conduction velocity are elevated at higher muscle temperatures during maximal power output development in humans. Am. J. Physiol. Regul. Integr. Comp. Physiol. 290, R376-R382. doi: 10.1152/ajpregu.00291.2005

Hamburg, N. M., and Balady, G. J. (2011). Exercise rehabilitation in peripheral artery disease: functional impact and mechanisms of benefits. Circulation 123, 87-97. doi: 10.1161/CIRCULATIONAHA.109.881888

Hammond, R. L., Augustyniak, R. A., Rossi, N. F., Lapanowski, K., Dunbar, J. C., and O'Leary, D. S. (2001). Alteration of humoral and peripheral vascular responses during graded exercise in heart failure. J. Appl. Physiol. 90, 55-61. doi: 10.1152/jappl.2001.90.1.55

Jeong, S. H., Hanh, T. M., Kim, H. K., Lee, S. R., Song, I. S., Noh, S. J., et al. (2013). HS-1793, a recently developed resveratrol analogue protects rat heart against hypoxia/reoxygenation injury via attenuating mitochondrial damage. Bioorg. Med. Chem. Lett. 23, 4225-4229. doi: 10.1016/j.bmcl.2013.05.010 recording, and drafted the manuscript. LJ designed experiments, oversaw performance of the experiments and data analysis, and revised the manuscript. All authors approved the final version of the article submitted for publication.

\section{FUNDING}

This study was supported by NIH P01 HL134609 and R01 HL141198.

\section{ACKNOWLEDGMENTS}

The authors greatly thank Chunying Yang for the excellent technical assistance.

Kaufman, M. P., and Forster, H. V. (1996). "Reflexes controlling circulatory, ventilatory and airway responses to exercise. Chapter 10" in Handbook of physiology-section 12: Exercise: Regulation and integration of multiple systems. eds. L. B. Rowell and J. T. Shepherd (New York: Oxford University Press), 381-447.

Kluess, H. A., Buckwalter, J. B., Hamann, J. J., and Clifford, P. S. (2005). Elevated temperature decreases sensitivity of $\mathrm{P} 2 \mathrm{X}$ purinergic receptors in skeletal muscle arteries. J. Appl. Physiol. 99, 995-998. doi: 10.1152/japplphysiol. 00319.2005

Li, J., King, N. C., and Sinoway, L. I. (2005). Interstitial ATP and norepinephrine concentrations in active muscle. Circulation 111, 2748-2751. doi: 10.1161/ CIRCULATIONAHA.104.510669

Li, J., and Xing, J. (2012). Muscle afferent receptors engaged in augmented sympathetic responsiveness in peripheral artery disease. Front. Physiol. 3:247. doi: $10.3389 /$ fphys.2012.00247

Liu, J., Li, J. D., Lu, J., Xing, J., and Li, J. (2011). Contribution of nerve growth factor to upregulation of $\mathrm{P} 2 \mathrm{X}(3)$ expression in DRG neurons of rats with femoral artery occlusion. Am. J. Physiol. Heart Circ. Physiol. 301, H1070-H1079. doi: 10.1152/ajpheart.00188.2011

Lu, J., Xing, J., and Li, J. (2013). Bradykinin B2 receptor contributes to the exaggerated muscle mechanoreflex in rats with femoral artery occlusion. Am. J. Physiol. Heart Circ. Physiol. 304, H1166-H1174. doi: 10.1152/ ajpheart.00926.2012

Ma, B., Yu, L. H., Fan, J., Cong, B., He, P., Ni, X., et al. (2011). Estrogen modulation of peripheral pain signal transduction: involvement of $\mathrm{P}_{2} \mathrm{X}_{3}$ receptors. Purinergic Signal 7, 73-83. doi: 10.1007/s11302-010-9212-9

McCloskey, D. I., and Mitchell, J. H. (1972). Reflex cardiovascular and respiratory responses originating in exercising muscle. J. Physiol. 224, 173-186. doi: 10.1113/jphysiol.1972.sp009887

McDermott, M. M., Liu, K., Greenland, P., Guralnik, J. M., Criqui, M. H., Chan, C., et al. (2004). Functional decline in peripheral arterial disease: associations with the ankle brachial index and leg symptoms. JAMA 292, 453-461. doi: 10.1001/jama.292.4.453

Mitchell, J. H., Kaufman, M. P., and Iwamoto, G. A. (1983). The exercise pressor reflex: its cardiovascular effects, afferent mechanisms, and central pathways. Annu. Rev. Physiol. 45, 229-242. doi: 10.1146/annurev.ph.45.030183.001305

Neff, D., Kuhlenhoelter, A. M., Lin, C., Wong, B. J., Motaganahalli, R. L., and Roseguini, B. T. (2016). Thermotherapy reduces blood pressure and circulating endothelin-1 concentration and enhances leg blood flow in patients with symptomatic peripheral artery disease. Am. J. Physiol. Regul. Integr. Comp. Physiol. 311, R392-R400. doi: 10.1152/ajpregu.00147.2016

Paudel, H. K., and Carlson, G. M. (1991). The ATPase activity of phosphorylase kinase is regulated in parallel with its protein kinase activity. J. Biol. Chem. 266, 16524-16529.

Prior, B. M., Lloyd, P. G., Ren, J., Li, H., Yang, H. T., Laughlin, M. H., et al. (2004). Time course of changes in collateral blood flow and isolated vessel 
size and gene expression after femoral artery occlusion in rats. Am. J. Physiol. Heart Circ. Physiol. 287, H2434-H2447. doi: 10.1152/ajpheart.00398.2004

Qin, L., Li, Q., and Li, J. (2020). Heat treatment improves the exaggerated exercise pressor reflex in rats with femoral artery occlusion via a reduction in the activity of the P2X receptor pathway. J. Physiol. 598, 1491-1503. doi: $10.1113 / J P 279230$

Rutkove, S. B., Kothari, M. J., and Shefner, J. M. (1997). Nerve, muscle, and neuromuscular junction electrophysiology at high temperature. Muscle Nerve 20, 431-436. doi: 10.1002/(SICI)1097-4598(199704)20:4<431::AID-MUS5> 3.0.CO;2-B

Shoemaker, J. K., Naylor, H. L., Hogeman, C. S., and Sinoway, L. I. (1999). Blood flow dynamics in heart failure. Circulation 99, 3002-3008. doi: 10.1161/01.CIR.99.23.3002

Simao, F., Matte, A., Matte, C., Soares, F. M., Wyse, A. T., Netto, C. A., et al. (2011). Resveratrol prevents oxidative stress and inhibition of $\mathrm{Na}^{+} \mathrm{K}^{+}$-ATPase activity induced by transient global cerebral ischemia in rats. J. Nutr. Biochem. 22, 921-928. doi: 10.1016/j.jnutbio.2010.07.013

Sinoway, L., Prophet, S., Gorman, I., Mosher, T., Shenberger, J., Dolecki, M., et al. (1989). Muscle acidosis during static exercise is associated with calf vasoconstriction. J. Appl. Physiol. 66, 429-436. doi: 10.1152/jappl.1989.66.1.429

Smith, S. A., Mitchell, J. H., and Garry, M. G. (2001). Electrically induced static exercise elicits a pressor response in the decerebrate rat. J. Physiol. 537, 961-970. doi: 10.1113/jphysiol.2001.012918

Song, P., Rudan, D., Zhu, Y., Fowkes, F. J. I., Rahimi, K., Fowkes, F. G. R., et al. (2019). Global, regional, and national prevalence and risk factors for peripheral artery disease in 2015: an updated systematic review and analysis. Lancet Glob. Health 7, e1020-e1030. doi: 10.1016/S2214-109X(19) 30255-4

Stewart, K. J., Hiatt, W. R., Regensteiner, J. G., and Hirsch, A. T. (2002). Exercise training for claudication. N. Engl. J. Med. 347, 1941-1951. doi: 10.1056/NEJMra021135

Thomas, K. N., van Rij, A. M., Lucas, S. J., and Cotter, J. D. (2017). Lower-limb hot-water immersion acutely induces beneficial hemodynamic and cardiovascular responses in peripheral arterial disease and healthy, elderly controls. Am. J. Physiol. Regul. Integr. Comp. Physiol. 312, R281-R291. doi: 10.1152/ajpregu.00404.2016

Tsuzuki, K., Kondo, E., Fukuoka, T., Yi, D., Tsujino, H., Sakagami, M., et al. (2001). Differential regulation of P2X(3) mRNA expression by peripheral nerve injury in intact and injured neurons in the rat sensory ganglia. Pain 91, 351-360. doi: 10.1016/S0304-3959(00)00456-5

Vanoli, E., and Schwartz, P. J. (1990). Sympathetic--parasympathetic interaction and sudden death. Basic Res. Cardiol. 85, 305-321.

Victor, R. G., Bertocci, L., Pryor, S., and Nunnally, R. (1988). Sympathetic nerve discharge is coupled to muscle cell $\mathrm{pH}$ during exercise in humans. J. Clin. Invest. 82, 1301-1305. doi: 10.1172/JCI113730

Waters, R. E., Terjung, R. L., Peters, K. G., and Annex, B. H. (2004). Preclinical models of human peripheral arterial occlusive disease: implications for investigation of therapeutic agents. J. Appl. Physiol. 97, 773-780. doi: 10.1152/ japplphysiol.00107.2004

Wilson, J. R., and Mancini, D. M. (1993). Factors contributing to the exercise limitation of heart failure. J. Am. Coll. Cardiol. 22, 93A-98A.

Xing, J., Lu, J., and Li, J. (2013). Augmented P2X response and immunolabeling in dorsal root ganglion neurons innervating skeletal muscle following femoral artery occlusion. J. Neurophysiol. 109, 2161-2168. doi: 10.1152/jn.01068.2012

Xing, J., Lu, J., and Li, J. (2018). Role of TNF-alpha in regulating the exercise pressor reflex in rats with femoral artery occlusion. Front. Physiol. 9:1461. doi: 10.3389/fphys.2018.01461

Ziegler, M. A., Distasi, M. R., Bills, R. G., Miller, S. J., Alloosh, M., Murphy, M. P., et al. (2010). Marvels, mysteries, and misconceptions of vascular compensation to peripheral artery occlusion. Microcirculation 17, 3-20. doi: 10.1111/ j.1549-8719.2010.00008.x

Ziganshin, A. U., Rychkov, A. V., Ziganshina, L. E., and Burnstock, G. (2002). Temperature dependency of $\mathrm{P} 2$ receptor-mediated responses. Eur. J. Pharmacol. 456, 107-114. doi: 10.1016/S0014-2999(02)02655-9

Conflict of Interest: The authors declare that the research was conducted in the absence of any commercial or financial relationships that could be construed as a potential conflict of interest.

Copyright (C) 2020 Qin and Li. This is an open-access article distributed under the terms of the Creative Commons Attribution License (CC BY). The use, distribution or reproduction in other forums is permitted, provided the original author(s) and the copyright owner(s) are credited and that the original publication in this journal is cited, in accordance with accepted academic practice. No use, distribution or reproduction is permitted which does not comply with these terms. 